\title{
Handheld laser-fiber vibrometry probe for assessing auditory ossicles displacement
}

\author{
Marcin Masalski $\odot,{ }^{\mathrm{a}, \mathrm{b}, *}$ Adam Wąż, ${ }^{\mathrm{c}}$ Przemysław Błauciak, ${ }^{\mathrm{d}}$ \\ Tomasz Zatoński, ${ }^{a}$ and Krzysztof Morawski ${ }^{\mathrm{e}}$ \\ ${ }^{a}$ Wroclaw Medical University, Department of Otolaryngology Head and Neck Surgery, \\ Wroclaw, Poland \\ ${ }^{b}$ Wroclaw University of Science and Technology, Department of Biomedical Engineering, \\ Wroclaw, Poland \\ ${ }^{c}$ Wroclaw University of Science and Technology, Department of Field Theory, \\ Electronic Circuits, and Optoelectronics, Wroclaw, Poland \\ ${ }^{\mathrm{d}}$ Wroclaw Medical University, Department of Neurosurgery, Wroclaw, Poland \\ ${ }^{\mathrm{e}}$ University of Opole, Institute of Medical Sciences, Opole, Poland
}

\begin{abstract}
Significance: Measurements of auditory ossicles displacement are commonly carried out by means of laser-Doppler vibrometry (LDV), which is considered to be a gold standard. The limitation of the LDV method, especially for in vivo measurements, is the necessity to expose an object in a straight line to a laser beam operating from a distance. An alternative to this approach is the use of a handheld laser-fiber vibrometry probe (HLFVP) with a curved tip.

Aim: We evaluate the feasibility of an HLFVP with a curved tip for measuring sound-induced displacement of the auditory ossicles.

Approach: A handheld vibrometer probe guiding the laser beam with a fiber-optic cable was used for displacement measurements of the incus body and the posterior crus of the stapes. Tonal stimuli at frequencies of $0.5,1,2$, and $4 \mathrm{kHz}$ were presented by means of an insert earphone positioned in the outer ear canal. The probe was fixed at the measurement site using a tripod or hand-held by one of the two surgeons.

Results: The measurements were carried out on six fresh temporal bones. Multivariate analysis of variance showed statistically significant differences for stimulus frequency $\left(F_{3,143}=29.37\right.$, $p<0.001$, and $\left.\eta^{2}=0.35\right)$, bone $\left(F_{5.143}=4.61, p=0.001\right.$, and $\left.\eta^{2}=0.01\right)$, and measurement site $\left(F_{1,143}=4.74, p=0.03\right.$, and $\left.\eta^{2}=0.02\right)$ in the absence of statistically significant differences for the probe fixation method $\left(F_{2,143}=0.15, p=0.862\right.$, and $\left.\eta^{2}=0.001\right)$. Standard deviations of the means were $6.9,2.6,1.9$, and $0.6 \mathrm{~nm} / \mathrm{Pa}$ for frequency, bone, site, and fixation, respectively. Ear transfer functions were found to be consistent with literature data.

Conclusions: The feasibility of applying HLFVP to measure the displacement of auditory ossicles has been confirmed. HLFVP offers the possibility of carrying out measurements at various angles; however, this needs to be standardized taking into account anatomical limitations and surgical convenience.

(C) The Authors. Published by SPIE under a Creative Commons Attribution 4.0 Unported License. Distribution or reproduction of this work in whole or in part requires full attribution of the original publication, including its DOI. [DOI: 10.1117/1.JBO.26.7.077001]
\end{abstract}

Keywords: laser-fiber vibrometer; handheld probe; middle ear mechanics; middle ear surgery; laser-Doppler vibrometer.

Paper 210077RR received Mar. 9, 2021; accepted for publication Jun. 17, 2021; published online Jul. 21, 2021; corrected Jul. 22, 2021.

*Address all correspondence to Marcin Masalski, marcin.masalski@pwr.edu.pl 


\section{Introduction}

Laser-Doppler vibrometry (LDV) is widely used in the assessment of auditory ossicles displacement. The method has been used in fundamental research on middle ear mechanics, ${ }^{1-6}$ optimization of surgical procedures ${ }^{2,7-15}$ such as intraoperative qualification for implantable middle ear hearing devices, ${ }^{2,16,17}$ and intraoperative evaluation of hearing improvement during ossiculoplasty. ${ }^{18-26}$ Vibrometric studies have also been conducted for potential applications in the clinical diagnosis of hearing loss. ${ }^{27-30}$ The LDV method enables non-contact measurement in a wide frequency band at a precisely determined point and is a gold standard in cadaver tests. However, despite numerous in vivo studies, it is not routinely used in clinical settings.

One of the LDV's limitations is the requirement to access the measurement site in a straight line. This often prevents reaching middle ear elements without damaging functionally relevant structures, especially since the laser beam must also bypass the eardrum, which collects the sound stimulus. In particular, these limitations apply to the measurement of the stapedial footplate, which is the last element of the middle ear's conductive chain. With a standard surgical approach to the middle ear cavity via a posterior tympanotomy, the access to the footplate is often limited by the facial nerve canal. In cadaver studies, the approach is extended by removing the canal and opening the facial recess, ${ }^{7,8,11,31,32}$ which cannot be done in vivo due to facial nerve palsy. In vivo measurement must be carried out on the posterior crus of the stapes ${ }^{1,3,15,17,24,31}$ or prosthesis in the case of crus damage. ${ }^{19-22}$ The transcanal approach consisting in elevation of the tympanic membrane is possible for vibrometry measurement using an alternative electromagnetic stimulation by means of a coil placed on the manubrium of the malleus. ${ }^{10,23}$ Nonetheless, the measuring site must still be within a straight line to the LDV device.

The development of endoscopic middle ear surgery techniques offering wider views and access to the areas of middle ear unreachable by microscopes ${ }^{33-36}$ entails the need to develop a vibrometric probe that can overcome the limitations of the straight-line access to the measurement site. Additionally, due to the high costs of LDV, alternative solutions in the form of handheld probes have been investigated. ${ }^{37-42}$ Handheld probes measure the displacement of movable tips that are excited by touching the measurement site. Displacement can be registered by piezoelectric sensors, ${ }^{37,40}$ strain gauge, ${ }^{39}$ electromagnetic, ${ }^{38}$ electromechanical, ${ }^{42}$ or fiberoptic Fabry-Perot strain sensors. ${ }^{41}$ The general problem of touch probes is tremor leading to high pretension and stiffening of the measuring site. This phenomenon requires the use of additional control systems, e.g., hydraulic ${ }^{39}$ or magnetic ones. ${ }^{42}$

This paper presents a handheld laser-fiber vibrometry probe (HLFVP) that guides the laser beam to the measurement site with a fiber-optic cable, thus allowing the access to spots unreachable by the laser beam of standard LDV device, while maintaining the advantages of non-contact measurement. ${ }^{43}$ The aim of this study is to evaluate the feasibility of using the HLFVP in the measurement of the auditory ossicles displacement and to compare the results with data reported in the literature.

The concept of the HLFVP is to illuminate a vibrating object with a laser beam coming out of an optical fiber and to collect diffused laser beam shifted in the frequency domain by the Doppler effect to a second optical fiber. The displacement is calculated based on the interference of the diffused laser beam with the reference beam by means of phase detection and demodulation. Standard LDV uses He-Ne gas lasers with a wavelength of $632.8 \mathrm{~nm}$, which is subject to significant limitations when guided by an optical fiber. The use of the semiconductor laser diode with a wavelength of $1550 \mathrm{~nm}$ allowed for applying fiber-optic technology to developing the laser-fiber Doppler vibrometer. Since the light at a wavelength of $1550 \mathrm{~nm}$ is invisible to the human eye, a navigation spot of red light is introduced to mark the site. ${ }^{43}$

Displacement measurements depend on the site and approach to the site, which is directly related to the measurement angle between the laser beam and the direction of movement. ${ }^{3,31}$ Relatively small differences are related to the type of material: in vivo, fresh, or frozen temporal bones. ${ }^{44,45}$ To compare the study results with the data available in the literature, ${ }^{1-3,12,16,17,31}$ measurements were performed on the posterior crus of the stapes and the incus body using the surgical approach via posterior tympanotomy with the facial nerve preservation. In this study, fresh cadaver specimens were used. 


\section{Materials and Methods}

The study was carried out in the Dissection Room of the University Clinical Hospital during the anatomopathological examination. The consent to conduct the trial was granted by the Bioethics Committee of Wroclaw Medical University in accordance with the World Medical Association Declaration of Helsinki. Each time, the consent to the anatomopathological examination was issued according to the applicable legal basis.

\subsection{Material}

Tests were performed on cadaveric donors within $48 \mathrm{~h}$ of death. The cadaver was kept in a cold storage at $4.5^{\circ} \mathrm{C}$ and placed on a sectional table about $3 \mathrm{~h}$ prior to the measurements. The external auditory canal was examined otoscopically for the presence of a normal eardrum. Cerumen was removed if present. The ER-3A insert earphone (Etymotic Research, IL, USA) with foam ear-tip was placed in the external acoustic canal. After the behind-the-ear incision, a self-retaining Weitlaner retractor was positioned to hold the auricle forward, and the mastoid plane was exposed. A standard antromastoidectomy followed by posterior tympanotomy with preservation of the facial nerve canal and the chorda tympani was performed. The stapedius tendon was severed to improve access to the posterior crus of the stapes. Measurements were conducted directly after preparation.

\subsection{Sound Stimulus}

Tonal stimuli at frequencies of $500 \mathrm{~Hz}, 1,2$, and $4 \mathrm{kHz}$ were presented simultaneously using earphones driven by a programmable generator RIGOL DG1062Z (Rigol, OR, USA). The voltage on the generator was adjusted to match the voltage of the Interacoustic AD629 (Interacoustic, Denmark) audiometer calibrated in accordance with ISO 389-1:1998 for the same headphones as used in the measurements. The sound pressure level at the eardrum was determined based on the real-ear to dial difference (REDD) values ${ }^{46}$ (Table 1).

\subsection{Measurements}

The displacement of the auditory ossicles was measured using a four-channel laser fiber Doppler vibrometer (LFDV) developed by Laser and Fiber Electronics Group at Wroclaw University of Science and Technology [Fig. 1(a)]. Detailed information on the operation of this device is presented in the articles by Wąż et al. (2009) ${ }^{43}$ and Wąż et al. (2014). ${ }^{47}$ The most important parameters of the LFDV are presented in Table 2.

The measuring probe consisted of a ceramic ferrule with an external diameter of $1.25 \mathrm{~mm}$ mounted inside a metal shielding sleeve [Fig. 1(b)]. Three standard single-mode telecommunications optical fibers with a jacket diameter of $125 \mu \mathrm{m}$ were glued into the ferrule. The diameter of the measuring spot coming out of the optical fiber is a function of the distance between the probe tip and the object, and for $1.5 \mathrm{~mm}$, it is about $300 \mu \mathrm{m}$. The diameter of the navigation spot was slightly larger because a telecommunications optical fiber at this wavelength (about $635 \mathrm{~nm}$ ) is not single mode. The metal tube was bent at $7 \mathrm{deg}$ with a $50-\mathrm{mm}$ radius of the curvature.

The response was recorded in sweeps of $200 \mathrm{~ms}$. The quadrature demodulator as a displacement decoder was used. ${ }^{47}$ Sweeps exceeding the heuristically determined noise threshold were rejected from the analysis. ${ }^{48}$ The baseline-to-peak amplitude displacement was determined from a 6-s long-response signal consisting of 30 sweeps. Figure 2 shows an example of the data obtained during the measurements against a background noise.

Table 1 REDD values for ER-3A earphones with foam ear-tip by Munro and Lazenby. ${ }^{46}$

\begin{tabular}{lcccc}
\hline \hline Frequency (Hz) & 500 & 1000 & 2000 & 4000 \\
REDD (dB) (SD) & $9.6(1.6)$ & $5.7(1.8)$ & $11.9(1.7)$ & $8.8(1.9)$ \\
\hline \hline
\end{tabular}




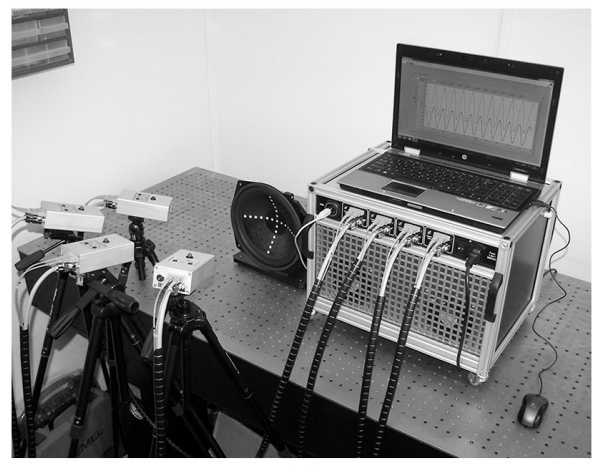

(a)

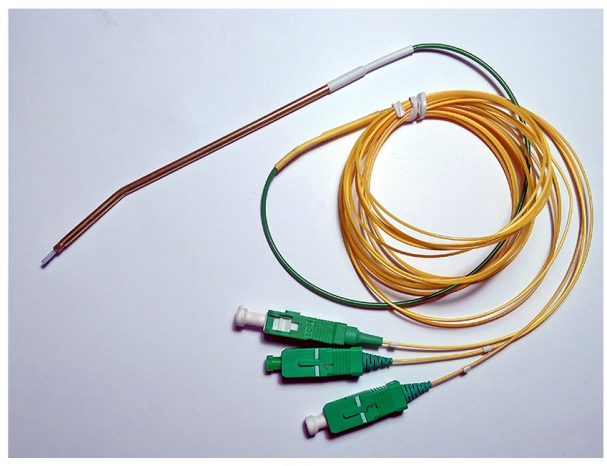

(b)

Fig. 1 (a) The four channel laser-fiber Doppler vibrometer ${ }^{47}$ and (b) the handheld probe. The probe, consisting of a ceramic ferrule with the three optical fibers mounted inside a metal shielding sleeve, was connected to one of the four channels of the laser-fiber vibrometer. Two optical fibers are for the forward and return paths of the $1550 \mathrm{~nm}$ laser, and one is for the red navigation spot.

Table 2 Parameters of the four-channels laser fiber Doppler vibrometer. ${ }^{47}$

\begin{tabular}{|c|c|}
\hline Parameter & Value \\
\hline Number of channels & 4 \\
\hline Wavelengths of the analyzing radiation $(\mathrm{nm})$ & $1549.32,1550.12,1550.92$, and 1551.72 \\
\hline Range of measured vibration speeds & $0 \div 3 \mathrm{~m} / \mathrm{s}$ \\
\hline Frequency of measured vibrations & $0.1 \mathrm{~Hz} \div 500 \mathrm{kHz}$ \\
\hline Resolution of displacement measurements & $10 \mathrm{pm}$ \\
\hline $\begin{array}{l}\text { Dynamic range (displacement measurement) } \\
\text { at } 1 \mathrm{kHz}\end{array}$ & $70 \mathrm{pm} \div 400 \mu \mathrm{m}$ \\
\hline Input optical power & $-40 \div 10 \mathrm{dBm}$ \\
\hline Number of phase/frequency demodulators & $\begin{array}{l}1 \times \text { phase demodulator (displacement), } 2 \times \mathrm{FM} \\
\text { demodulators (velocity) }\end{array}$ \\
\hline Measuring distance & $1 \mathrm{~mm} \div 2.5 \mathrm{~m}$ (depending on the probe) \\
\hline Noise & Fig. 2 \\
\hline $\begin{array}{l}\text { Auxiliary laser radiation (observation of the } \\
\text { analysis point) }\end{array}$ & $635 \mathrm{~nm}(\mathrm{red})$ \\
\hline Software for control and acquisition & LabView \\
\hline
\end{tabular}

A small piece of reflective tape was placed on the incus body and the posterior crus of the stapes. The measurement at each site was carried out three times. The probe was held by one of the two surgeons two times; the third time the probe was placed on a tripod. The intensities of all four tonal stimuli were equal and were set to $90 \mathrm{~dB}$ HL.

Measurements on the posterior crus of the stapes were also carried out in relation to the intensities of the sound stimuli. The intensities were decreased from $90 \mathrm{~dB}$ HL in $10 \mathrm{~dB}$ steps until no response was obtained. The probe was fixed on a tripod.

\subsection{Statistical Analysis}

The measurements were compared with data reported in the literature on the basis of confidence intervals on the difference between means. Multivariate analysis of variance (MANOVA) was 


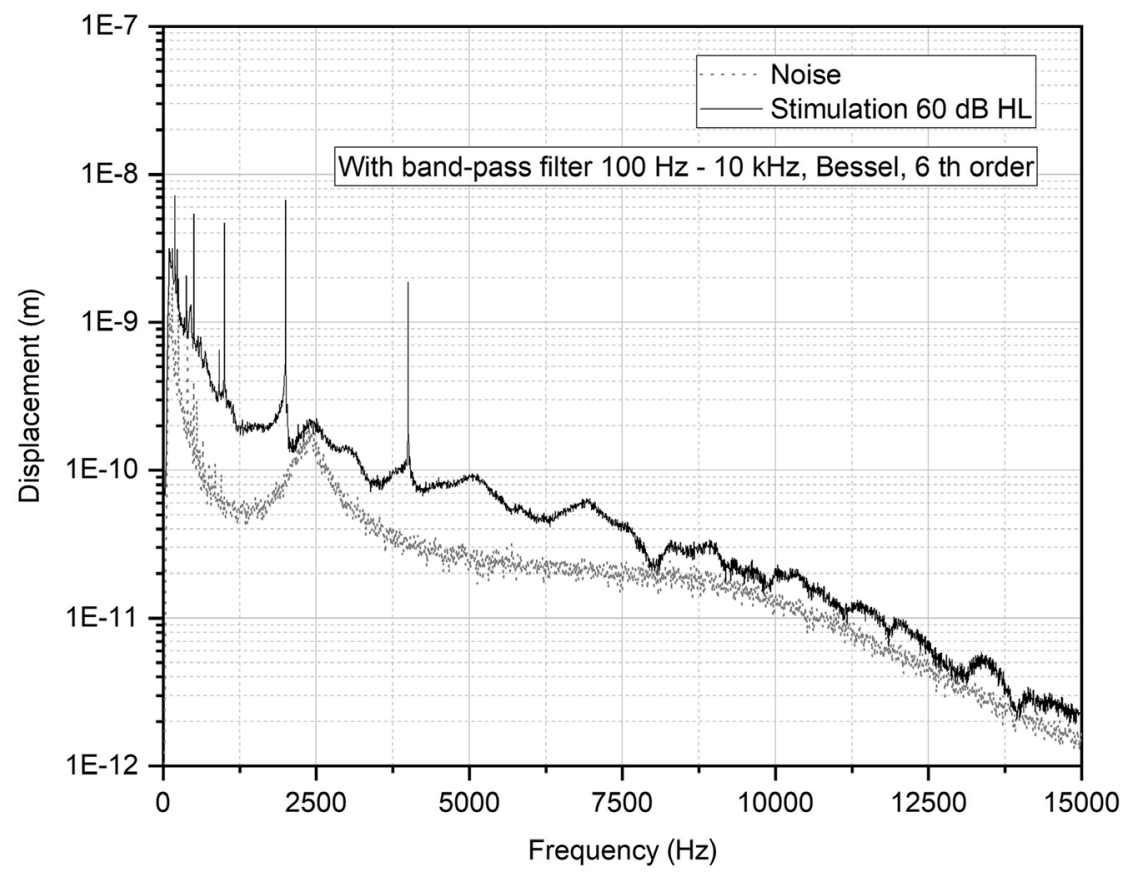

Fig. 2 Noise floor (gray dots) measured on a stationary object and the displacement of the posterior crus of the stapes with a stimulation of $60 \mathrm{~dB} \mathrm{HL}$ (black line). The probe was fixed on a tripod.

carried out for stimulus frequency, bone, site, and fixation method (operators/tripod). Pearson correlation coefficients between the logarithm of the displacement and the stimulus intensity were determined.

\section{Results}

In the period from 25.06.2019 to 23.01.2020, measurements were performed on 6 out of 7 temporal bones from 6 donors aged 48 to 68 years (median 62, SD 7). All of the ears appeared normal on otoscopic inspection; however, during mastoidectomy of the temporal bone no. 2 , inflammatory changes were found in the tympanic cavity, and this bone was excluded from further analysis. A photograph of the temporal bone with reflective material at the sites and the second photo taken during the measurement on the posterior crus of the stapes are shown in Fig. 3.

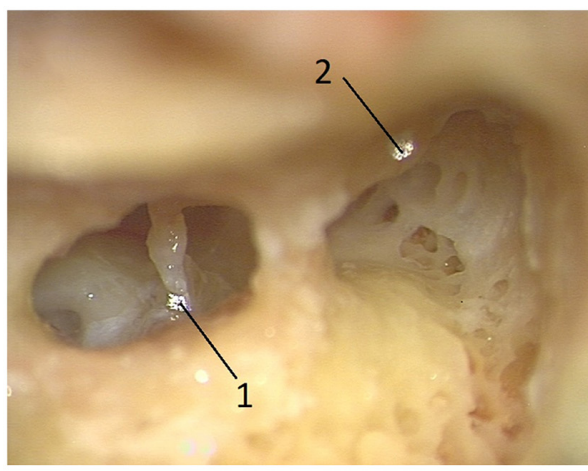

(a)

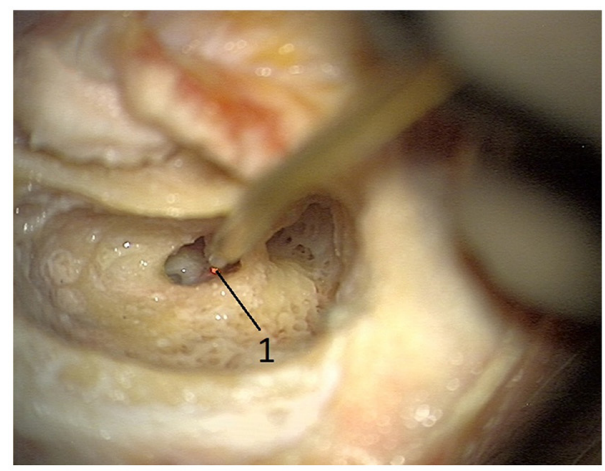

(b)

Fig. 3 Photograph of the temporal bone with reflective material (a) placed at the measuring sites: on the posterior crus of the stapes (1) and the incus body (2). The approach to the tympanic cavity was performed by means of the standard antromastoidectomy followed by posterior tympanotomy with preservation of the facial nerve canal and the chorda tympani. (b) Photograph of the measurement on the posterior crus of the stapes (1). The probe was introduced into the tympanic cavity through a posterior tympanotomy. 
The middle ear transfer functions were calculated for measurements on the posterior crus of the stapes and the incus body as well as for individual temporal bones and regarding the probe fixation method (Fig. 4, Table S1 in the Supplementary Material). The results were compared with data reported in the literature. ${ }^{1-3,12,16,17,31}$ Statistically significant differences at $p<0.05$ were obtained only for the study by Seidman et al. ${ }^{17}$ and only for the measurement at $500 \mathrm{~Hz}$ on the incus body [Fig. 4(b)]. The difference between the means was at the level of $62.1 \mathrm{~nm} / \mathrm{Pa}$.

The data were subjected to MANOVA. Statistically significant differences were obtained for frequency $\left(F_{3,143}=29.37, p<0.001\right.$, and $\left.\eta^{2}=0.35\right)$, bone $\left(F_{5,143}=4.61, p=0.001\right.$, and $\left.\eta^{2}=0.01\right)$, and measurement site $\left(F_{1,143}=4.74, p=0.03\right.$, and $\left.\eta^{2}=0.02\right)$ in the absence of statistically significant differences for the probe fixation method $\left(F_{2,143}=0.15, p=0.862\right.$, and $\eta^{2}=0.001$ ). Standard deviations of the means were $6.9,2.6,1.9$, and $0.6 \mathrm{~nm} / \mathrm{Pa}$ for frequency, bone, site, and fixation, respectively. Test-retest reliability was assessed by means of intersurgeon differences. The standard deviation of the intersurgeon differences was determined at the level of $3.26 \mathrm{~nm} / \mathrm{Pa}$ with a Cronbach alpha at 0.96 (95\% CI 0.93 to 0.98 ).

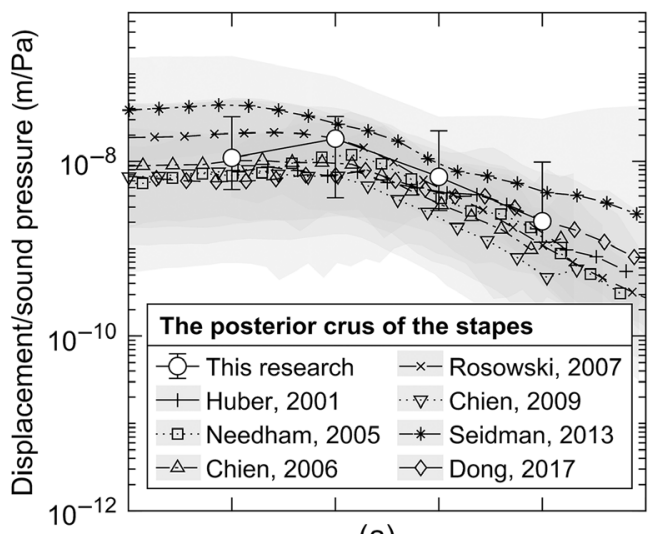

(a)

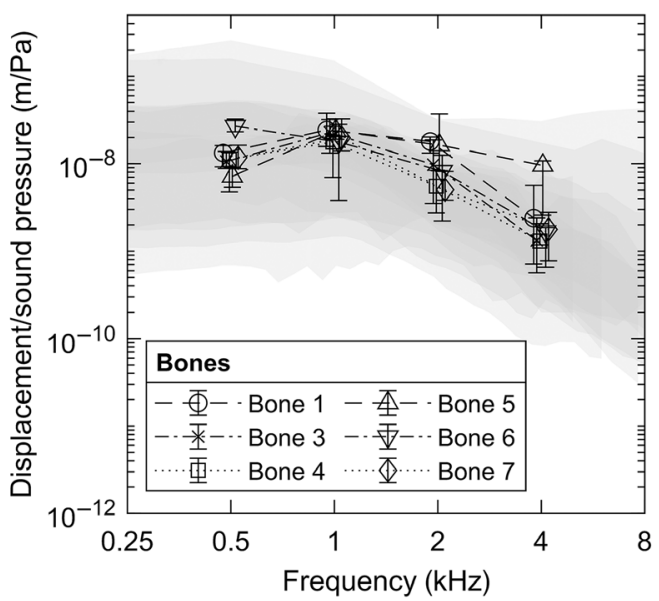

(c)

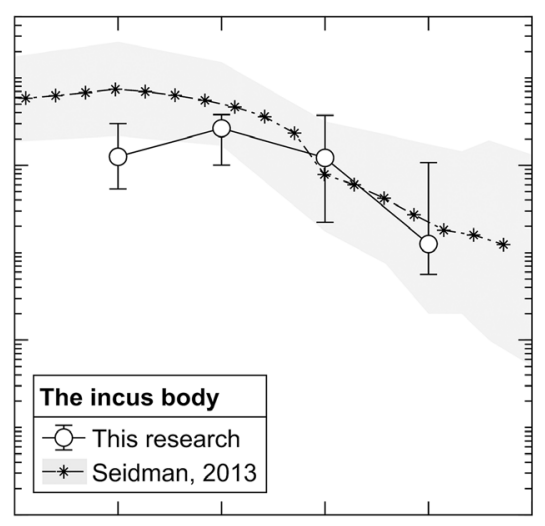

(b)

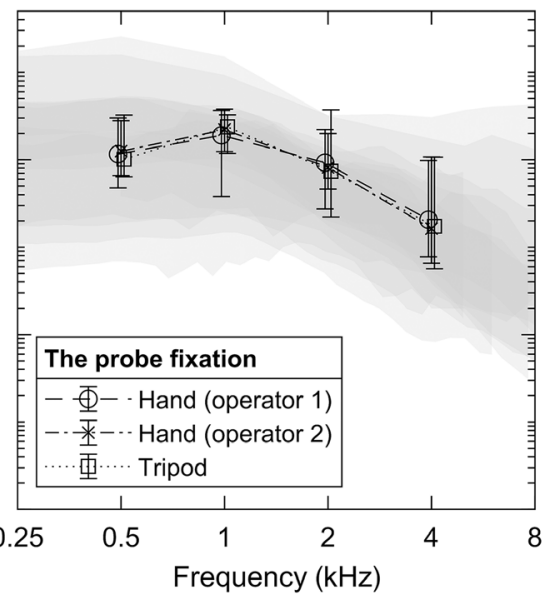

(d)

Fig. 4 The middle ear transfer functions. Single data point represents the interpolated median of 18 measurements of the posterior crus of the (a) stapes and (b) incus body taken on six temporal bones by two surgeons and using a tripod. 95\% confidence intervals determined using bootstrapping are marked with whiskers. The gray areas present $95 \%$ confidence intervals reported in the literature. A slight horizontal shift at the frequencies $500 \mathrm{~Hz}, 1,2$, and $4 \mathrm{kHz}$ was introduced to improve symbol visualization. The article by Rosowski et al. ${ }^{16}$ presented an average of 13 other studies. The measurements in the research by Nedham et al. ${ }^{2}$ were carried out on the stapes head. The analogous chart for (c) temporal bone and (d) the fixation method is presented by means of the interpolated median of 6 and 12 ( 6 bones $\times 2$ sites) measurements, respectively. 


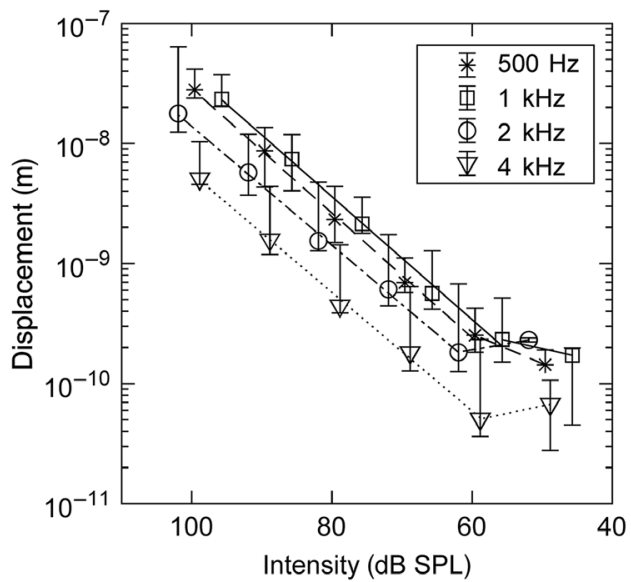

(a)

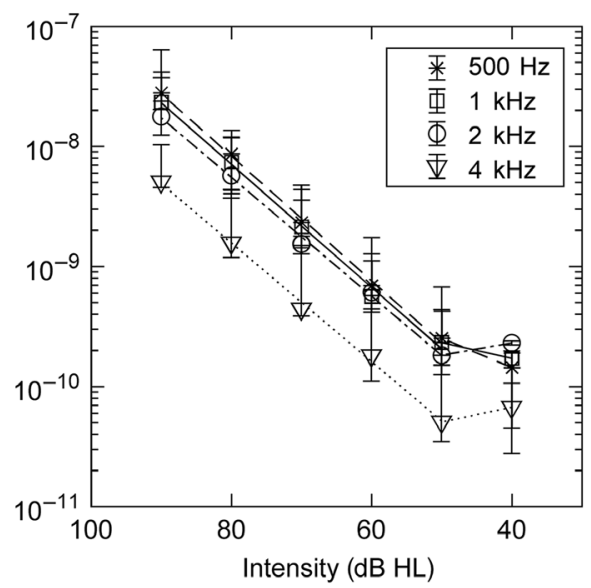

(b)

Fig. 5 The displacement of the posterior crus of the stapes in relation to the intensity of the sound stimulus in (a) dB SPL and (b) dB HL. Single data point presents interpolated median of 6 measurements. Whiskers present $95 \%$ confidence intervals determined by means of bootstrapping.

The displacement of the posterior crus of the stapes in the relation to the stimulus intensity (Table S2 in the Supplementary Material) is shown in Fig. 5. The logarithm of the displacement decreases linearly with a decrease in the stimulus intensity in the range up to $50 \mathrm{~dB}$ HL. Pearson's correlation coefficients were determined at the levels of $0.91,0.94,0.91$, and 0.87 for the frequencies $0.5,1,2$, and $4 \mathrm{kHz}$, respectively. The noise floor was present at the mean levels of $383 \mathrm{pm}$ (SD 207), $251 \mathrm{pm}$ (SD 43), $206 \mathrm{pm}$ (SD 55), and $51 \mathrm{pm}$ (SD 17) at frequencies $0.5,1,2$, and $4 \mathrm{kHz}$, respectively.

\section{Discussion}

Measurements of the auditory ossicles displacement evoked by a sound stimulus were carried out by means of an HLFVP. The results were compared with data reported in the literature ${ }^{1-3,12,16,17,31}$ obtaining high consistency.

No statistically significant differences at the level of $p<0.05$ were obtained between hand and tripod measurement. This indicates that tremor has negligible influence on the results. The observation is in line with expectations as the physiological tremor ranging from 8 to $12 \mathrm{~Hz}^{49}$ is significantly different from the frequency of the ossicles vibration.

A linear relation between the displacement logarithm and the stimulus intensity was obtained at $50 \mathrm{~dB} \mathrm{HL}$ and above. The threshold at $50 \mathrm{~dB} \mathrm{HL}$ was found to be significantly higher than the value determined intraoperatively at $21.6 \mathrm{~dB} \mathrm{HL}$ (SD 4.6). ${ }^{21}$ The difference may be related to the duration of the measurement as well as methodological differences in the assessment of the response threshold, which in the case of one study ${ }^{21}$ was evaluated subjectively in the time domain. However, the method of threshold assessment is of secondary importance due to the linearity of the process and the possibility of estimating the threshold from measurements at higher intensities.

The displacement measured by means of laser vibrometry is strongly dependent on the measuring angles between the laser beam and the vibration direction ${ }^{31}$ and the reflecting surface (Fig. 6). Controlling the angles is necessary to improve measurement uncertainty, especially since angle ranges for a handheld probe are wider than for a long-distance measurement. An alternative approach for measuring angles would be to standardize the site and the angles taking into account anatomical limitations and surgical convenience. Due to the difficulty of measuring angles in the operating room, standardization seems to be more efficient.

In this work, the measurement uncertainty may also be affected by the setup method of stimulus intensity. The sound pressure values at the tympanic membrane were determined on the REDD basis for the ER-3a with a foam ear-tip. This method is less precise than using an 


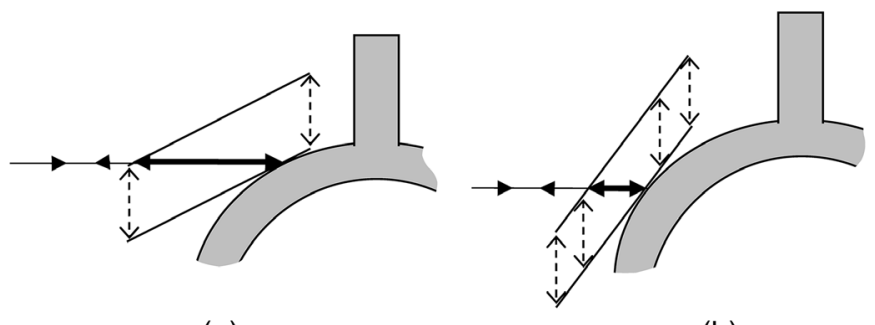

(a)

(b)

Fig. 6 Effects of the angle between the laser beam and the reflecting surface on displacement measurements. Both figures show the laser beam (full arrow) forming a right angle with the direction of stapes displacement (dashed arrow). The measured displacement (bold arrow) is greater in (a) than (b) as it depends on the angle between the laser and the reflective surface.

in-the-ear microphone such as ER-7, especially since the auricle was tilted forward and held in place by the self-retaining Weitlaner retractor, which could affect the ear canal geometry.

In this paper, the 1550-nm wavelength was used primarily because of compatibility with telecom, since optics and optical fibers for this wavelength are widespread and relatively inexpensive. At $1550 \mathrm{~nm}$, light scattering on dry bone was sufficient for measurement. However, on cadaveric bones, incoming body fluids changed the scattering to reflectance, which is more directional, and made it difficult for the signal to reach the receiving system. Therefore, it was necessary to apply reflective tape. Optimizing the wavelength in terms of the reflectance of moist bone can contribute to improved measurement quality.

The tip of the measuring probe had a diameter of $1.25 \mathrm{~mm}$. This dimension was related to the diameter of the shielding sleeve and the ferrule, into which the optical fibers were inserted. Theoretically, the diameter of the tip could be $<300 \mu \mathrm{m}$ and is limited by dimensions of the shielding sleeve for three optical fibers of $125 \mu \mathrm{m}$ each. The minimum bending radius of the probe tip associated with optical fiber bending losses is estimated at about $10 \mathrm{~mm}$.

\section{Conclusions}

The feasibility of using an HLFVP to measure the displacement of the auditory ossicles has been confirmed. HLFVP offers the possibility of carrying out measurements at various angles; however, this needs to be standardized taking into account anatomical limitations and surgical convenience. An additional advantage of the curved HLFVP tip in terms of access to the measurement site needs to be evaluated during the endoscopic approach.

\section{Disclosures}

The authors have no conflicts of interest to declare.

\section{Acknowledgments}

This work was supported by the statutory funds of Wroclaw Medical University.

\section{Code, Data, and Materials Availability}

The raw data are presented in Tables S1 and S2 in the Supplementary Material.

\section{References}

1. A. Huber et al., "Intraoperative assessment of stapes movement," Ann. Otol. Rhinol. Laryngol. 110(1), 31-35 (2001). 
2. A. J. Needham et al., "The effects of mass loading the ossicles with a floating mass transducer on middle ear transfer function," Otol. Neurotol. 26(2), 218-224 (2005).

3. W. Chien et al., "Measurements of stapes velocity in live human ears," Hear. Res. 249(1-2), 54-61 (2009).

4. M. Szymańskiv, "Vibrations of the human tympanic membrane measured with laser Doppler vibrometer," Otolaryngol. Pol. 63(2), 182-185 (2009).

5. S. Ihrle et al, "Biomechanics of the incudo-malleolar-joint: experimental investigations for quasi-static loads," Hear. Res. 340, 69-78 (2016).

6. P. K. Gottlieb, Y. Vaisbuch, and S. Puria, "Human ossicular-joint flexibility transforms the peak amplitude and width of impulsive acoustic stimuli," J. Acoust. Soc. Am. 143(6), 3418 (2018).

7. M. Asai, A. M. Huber, and R. L. Goode, "Analysis of the best site on the stapes footplate for ossicular chain reconstruction," Acta Otolaryngol. 119(3), 356-361 (1999).

8. D. P. Morris et al., "Optimum tension for partial ossicular replacement prosthesis reconstruction in the human middle ear," Laryngoscope 114(2), 305-308 (2004).

9. D. Jiang et al., "Equivalent noise level generated by drilling onto the ossicular chain as measured by laser Doppler vibrometry: a temporal bone study," Laryngoscope 117(6), 1040-1045 (2007).

10. J. Peacock, J. Dirckx, and M. von Unge, "Magnetically driven middle ear ossicles with laser vibrometry as a new diagnostic tool to quantify ossicular fixation," Acta Otolaryngol. 134(4), 352-357 (2014).

11. A. Niklasson et al., "Ossiculoplasty on isolated malleus fractures: a human temporal bone study using laser Doppler vibrometry," Otol. Neurotol. 37(7), 895-901 (2016).

12. W. Dong et al., "Middle-ear sound transmission under normal, damaged, repaired, and reconstructed conditions," Otol. Neurotol. 38(4), 577-584 (2017).

13. A. Niklasson et al., "An optimal partial ossicular prosthesis should connect both to the tympanic membrane and malleus: a temporal bone study using laser Doppler Vibrometry," Otol. Neurotol. 39(3), 333-339 (2018).

14. U. A. Gamm et al., "Optimum coupling of an active middle ear actuator: effect of loading forces on actuator output and conductive losses," Otol. Neurotol. 40(6), 789-796 (2019).

15. A. Rönnblom et al., "A new, promising experimental ossicular prosthesis: a human temporal bone study with laser Doppler vibrometry," Otol Neurotol. 41(4), 537-544 (2020).

16. J. J. Rosowski et al., "Testing a method for quantifying the output of implantable middle ear hearing devices," Audiol. Neurootol. 12(4), 265-276 (2007).

17. M. D. Seidman et al., "Normative data of incus and stapes displacement during middle ear surgery using laser Doppler vibrometry," Otol. Neurotol. 34(9), 1719-1724 (2013).

18. J. Sokołowski et al., "Method of ossicular chain valuation. Experimental measurement and clinical application," Otolaryngol. Pol. 63(5), $432-436$ (2009).

19. K. Niemczyk et al., "Ossiculoplasty efficacy monitored by ECoG-laser-Doppler technique," Otolaryngol. Head Neck Surg. 145(2_suppl), P222-P223 (2011).

20. K. F. Morawski et al., "Intraoperative monitoring of hearing improvement during ossiculoplasty by LDV-ABR-ECochG technique," Otolaryngol. Head Neck Surg. 149(2_suppl), P102-P103 (2013).

21. K. F. Morawski et al., "Intraoperative monitoring of hearing improvement during ossiculoplasty by laser-Doppler vibrometry, auditory brainstem responses, and electrocochleography," Otolaryngol. Head Neck Surg. 150(6), 1043-1047 (2014).

22. K. F. Morawski, K. Niemczyk, and R. Delgado, "Combined model of intraoperative monitoring of ossiculoplasty efficiency by laser-Doppler vibrometry and auditory evoked potentials," J. Laryngol. Otol. 130(S3), S16-S17 (2016).

23. T. Zahnert et al., "A new intraoperative real-time monitoring system for reconstructive middle ear surgery: an experimental and clinical feasibility study," Otol. Neurotol. 37(10), 1601-1607 (2016).

24. Y. Kunimoto et al, "Sequential motion of the ossicular chain measured by laser Doppler vibrometry," Acta Otolaryngol. 137(12), 1233-1237 (2017).

25. K. Niemczyk et al., "Objective assessment of hearing during second stage of tympanoplastysurgical technique and measurement methodology presented in ten patients," Clin. Otolaryngol. 43(1), 347-352 (2018). 
26. K. Gladiné et al, "Evaluation of artificial fixation of the incus and malleus with minimally invasive intraoperative laser vibrometry (MIVIB) in a temporal bone model," Otol. Neurotol. 41(1), 45-51 (2020).

27. J. J. Rosowski, R. P. Mehta, and S. N. Merchant, "Diagnostic utility of laser-Doppler vibrometry in conductive hearing loss with normal tympanic membrane," Otol. Neurotol. 24(2), 165-175 (2003).

28. W. Chien, J. J. Rosowski, and S. N. Merchant, "Investigation of the mechanics of type III stapes columella tympanoplasty using laser-Doppler vibrometry," Otol. Neurotol. 28(6), 782-787 (2007).

29. J. J. Rosowski, H. H. Nakajima, and S. N. Merchant, "Clinical utility of laser-Doppler vibrometer measurements in live normal and pathologic human ears," Ear Hear. 29(1), 3-19 (2008).

30. I. Arechvo et al., "Laser Doppler vibrometry of the middle ear in humans: derivation dependence, variability, and bilateral differences," Medicina 45(11), 878-886 (2009).

31. W. Chien et al., "The effect of methodological differences in the measurement of stapes motion in live and cadaver ears," Audiol. Neurootol. 11(3), 183-197 (2006).

32. M. Ryan et al., "Mechanical energy dissipation through the ossicular chain and inner ear using laser Doppler vibrometer measurement of round window velocity," Otol. Neurotol. 41(3), e387-e391 (2020).

33. E. B. Karchier et al., "Intraoperative endoscopy of the anterior epitympanum recess through the posterior tympanotomy," Wideochir. Inne Tech. Maloinwazyjne 11(3), 208-213 (2016).

34. S. Mitchell and C. Coulson, "Endoscopic ear surgery: a hot topic?" J. Laryngol. Otol. 131(2), 117-122 (2017).

35. S. Y. Han et al. "Comparison of endoscopic and microscopic ear surgery in pediatric patients: a meta-analysis," Laryngoscope 129(6), 1444-1452 (2019).

36. I. E. Emre et al., "Endoscopic ear surgery," J. Otol. 15(1), 27-32 (2020).

37. K. Gyo et al., "Assessment of stapes mobility by use of a newly developed piezoelectric ceramic device. A preliminary experiment in dogs," Ann. Otol. Rhinol. Laryngol. 109(5), 473-477 (2000).

38. T. Zahnert et al., "Intraoperative measurement of stapes mobility using a hand-guided electromagnetic probe," Laryngorhinootologie 80(2), 71-77 (2001).

39. T. Koike et al., "An apparatus for diagnosis of ossicular chain mobility in humans," Int. J. Audiol. 45(2), 121-128 (2006).

40. N. Hato et al., "A new tool for testing ossicular mobility during middle ear surgery: preliminary report of four cases," Otol. Neurotol. 27(5), 592-595 (2006).

41. T. T. Linder, G. Volkan, and E. Troxler, "Objective measurements of ossicular chain mobility using a palpating instrument intraoperatively," Otol. Neurotol. 36(10), 1669-1675 (2015).

42. D. Schwarz et al., "Evaluation of coupling efficiency in round window vibroplasty with a new handheld probe," Otol. Neurotol. 40(1), e40-e47 (2019).

43. A. T. Wąż, P. R. Kaczmarek, and K. M. Abramski, "Laser-fibre vibrometry at $1550 \mathrm{~nm}$," Meas. Sci. Technol. 20(10), 105301 (2009).

44. J. J. Rosowski et al., "Cadaver middle ears as models for living ears: comparisons of middle ear input immittance," Ann. Otol. Rhinol. Laryngol. 99(5 Pt 1), 403-412 (1990).

45. S. N. Merchant, M. R. Ravicz, and J. J. Rosowski, "Acoustic input impedance of the stapes and cochlea in human temporal bones," Hear. Res. 97(1-2), 30-45 (1996).

46. K. J. Munro and A. Lazenby, "Use of the "real-ear to dial difference' to derive real-ear SPL from hearing level obtained with insert earphones," Br. J. Audiol. 35(5), 297-306 (2001).

47. A. T. Wąż et al., "Multichannel WDM vibrometry at $1550 \mathrm{~nm}$," Photonics Lett. Pol. 6(4), 133-135 (2014).

48. D. G. Lee and P. P. Mercie, "Noise analysis of phase-demodulating receivers employing super-regenerative amplification," IEEE Trans. Microwave Theory Tech. 65(9), 3299-3311 (2017).

49. P. D. Charles et al., "Classification of tremor and update on treatment," Am. Fam. Phys. 59(6), 1565-1572 (1999). 
Marcin Masalski is an ENT specialist and computer scientist. He works as an assistant professor at Wroclaw Medical University, where he specializes in endoscopic sinus surgery and ear surgery. In addition to surgery, his interests include audiology research conducted through his position at the Wroclaw University of Science and Technology. His research has resulted in, inter alia, the popular hearing test mobile application for Android.

Adam Wąż works in the Laser and Fiber Electronics Group at Wroclaw University of Science and Technology. His main research interests are laser Doppler vibrometry: scattered light analysis, photodetection systems, demodulation, signal processing, and data acquisition. He is the author of the concept and control software of a four-channel laser-fiber Doppler vibrometer. He participates in research projects on laser vibrometry, fiber amplifiers, and ultrafast fiber lasers.

Przemysław Błauciak is a fellow doing residence training in neurosurgery and a student of $\mathrm{PhD}$ studies in the Neurosurgical Department of Wroclaw Medical University. He is a member of the Polish Society of Neurosurgeons. His main interests are skull base surgery, neuro-oncology, and endoscopy in neurosurgery.

Tomasz Zatoński is an associate professor and the head of the Otolaryngology-Head and Neck Surgery Department of Wroclaw Medical University, the vice-rector for the University Communications of Wroclaw Medical University, the chairman (elect 2022-2024) of the Polish Society of Otorhinolaryngologists Head and Neck Surgeons, and an expert in the field of otolaryngology for Lower Silesia Voivodship. He is the author of 100 papers (IF $=63.221$ and $\mathrm{H}$-index $=8,265$ citations).

Krzysztof Morawski is a professor of the Medical University of Opole, former professor of the Medical University of Warsaw and Wroclaw, otologist, neurotologist, and experimental audiologist; he was the Fulbright scholar in 2000/2001 at the University of Miami. His main interests are the intraoperative monitoring (IM) of hearing during acoustic tumor removal by ABR, DPOAEs, and ECochG, IM of ossiculoplasty efficiency by laser-Doppler vibrometry, and round-window ECochG. His previous research involves cochlear ischemia and prevention of irreversible cochlear damages investigated on animal models. 